\author{
Military Technical College \\ Kobry El-kobbah, \\ Cairo, Egypt
}

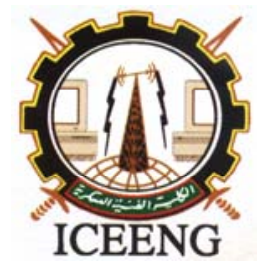
$5^{\text {th }}$ International Conference on Electrical Engineering ICEENG 2006

\title{
COMPUTER MODELING FOR NUCLEAR AND LIGHTNING ELECTROMAGNETIC PULSE (EMP) PROPAGATION AND COUPLING
}

\author{
Mohamed A. H. Eleiwa, Ph.D., \\ Egyptian Armed Forces
}

\begin{abstract}
This paper is primarily concerned with modeling EMP propagation and coupling to determine the external electromagnetic fields about, and hence the induced voltages and currents in complicated systems such as aircrafts, missiles, satellites, vehicles, buildings etc., under the illumination of Nuclear EMP (NEMP) or Lightning EMP (LEMP). Based on the transmission line (telegrapher) equations, a numerical procedure is developed to determine the distributed voltages and currents induced along the transmission lines exposed to NEMP and LEMP electromagnetic fields. The proposed procedure is applied to predict the EMP interaction with practical configurations such as a combat vehicle, over head power lines and telecommunication cables. The induced voltages are calculated and compared favorably with the published results, and hence the accuracy of the proposed procedure is proven. Such accurate coupling model is necessary for application of vulnerability assessment and protection techniques.
\end{abstract}

\section{KEY WORDS}

Electromagnetic Pulse, Coupling, and Computer Modeling

\section{INTRODUCTION}

Nuclear explosions in or above the earth's atmosphere produce high energy gamma rays. The intense pulse of gamma rays ionizes the air molecules producing free electrons. These free electrons are called Compton electrons, and their movement in relation to the earth's magnetic field is the source of this radiated electromagnetic energy known as electromagnetic pulse (NEMP) [1]. Nuclear detonations are normally grouped into three general classifications: surface bursts, endoatmospheric bursts, and exoatmospheric bursts. Exoatmospheric bursts (above about 70 miles) generate the high altitude EMP (HEMP). HEMP is the dominant concern in most hardness design issues. Electric fields as large as 50,000 volts/meter, with 10 to 20 nanosecond rise time pulses, and with pulse durations as long as one microsecond, have been estimated [2]. HEMP effects are especially damaging to integrated circuits and other sensitive low-voltage/current electronic devices on which 
facilities rely. Generally, there are two concepts to be considered as a methodology for HEMP protection: component or room hardening and facility shielding.

Lightning is basically a long spark that discharges regions of excess charge located in the atmosphere [3]. The total lightning discharge, called a flash, is initiated by a downward (or upward) traveling spark known as the stepped leader. Each flash between charge regions is made up of a number of independent current pulses called strokes. The nearby strikes generate radiated electromagnetic pulse, known as LEMP, while direct strike attachments actually involve pulsed current flow within an object or vehicle. Although less than two percent of all lightning strokes exceed 100 Kilo amperes, it has become the usual practice in industry to protect facilities against strokes at the 100 kilo amperes level. This protection normally consists of either a "zone of protection" for the structure, or of furnishing a conduction path on the structure to safely conduct the current to ground. The time waveform of the lightning stroke has a profound influence on the voltage conducted to ground on the systems conductors. Although actual generation mechanisms are different, the NEMP and LEMP threats can be addressed with similar analysis, test, and circuit protection techniques.

The problem of predicting the behavior of a complicated system in EMP environment may be simplified by considering small sub problems and analyzing them independently. Such sub problems are EMP phenomenology, propagation, coupling, transient analysis of driven circuits, and finally the overall system assessment. The total solution to the main problem is then looked upon as a combination of such solutions. Accurate evaluation of the coupling quantities (induced currents and voltages) is necessary to predict the system upset or failure from exposure to EM radiation. The transfer function describing the coupling depends, in general, on the frequency content, amplitude, and polarization of the incident electromagnetic waves. The coupling of an external EM fields to a line is generally evaluated using EM scattering theory [4]. However, in many cases of practical interest, simple transmission line models are sufficient. The three different approaches for solving the line coupling problem using the transmission line theory are Taylor [5], Agrawal [6], and Rachidi [7]. It is the purpose of this paper to study EMP coupling. A numerical procedure is therefore proposed to evaluate the induced currents and voltages in transmission lines exposed to different EMP waveforms. The radiated HEMP and LEMP electromagnetic fields are firstly formulated. The propagation parameters of incident, transmitted, and reflected waves from the earth are then determined, and hence the excitation fields are derived. Finally, the frequency and time-domain responses of the victim transmission lines are numerically calculated, and compared with the published results.

\section{THE WAVEFORMS AND RADIATED FIELDS OF NEMP AND LEMP}

The time-domain electric and magnetic fields of exoatmospheric burst NEMP waveform are given by Lee [2] as

$$
E(t)=E_{o}\left(e^{-\alpha t}-e^{-\beta t}\right), \quad H(t)=E(t) /(120 \pi)
$$

Where $\alpha$ and $\beta$ are constants to mimic the measured waveform. Generally, the NEMP waveform is characterized by the rise time $\boldsymbol{t}_{m}=\frac{\ln (\beta / \alpha)}{\beta-\alpha}$, the pulse width 


$$
\begin{aligned}
& \boldsymbol{t}_{o}=\frac{\beta-\alpha}{\alpha \beta}\left(e^{-\alpha t_{m}}-e^{-\beta t_{m}}\right)^{-1}, \text { and the peak value of the electric field } \\
& \boldsymbol{E}_{m}=\boldsymbol{E}_{o}\left(\boldsymbol{e}^{-\alpha t_{m}}-e^{-\beta t_{m}}\right)
\end{aligned}
$$

The lightning channel return current is modeled as an upward traveling current pulse plus its image in the ground plane, and given by [8] as

$$
\mathbf{i}(\mathbf{t}, \mathbf{z})=\mathbf{i}(\tau) \mathbf{e}^{-\alpha_{\mathrm{c}} \mathbf{z}}
$$

Where the retarded time $\tau=\boldsymbol{t}-\boldsymbol{z} / \boldsymbol{v}$, channel attenuation constant $=\boldsymbol{\alpha}_{\mathfrak{c}}$, and the velocity of propagation of lightning current pulse is $\boldsymbol{v}$. According to lightning measurements, the channel base current $\boldsymbol{i}(\boldsymbol{t})$ is expressed as the sum of two (Heidler) functions as

$i(t)=\sum_{m=1}^{m=2} \frac{I_{o_{m}}}{\eta_{m}} \frac{\left(t / \tau_{m_{1}}\right)^{n_{m}}}{1+\left(t / \tau_{m_{1}}\right)^{n_{m}}} e^{-t / \tau_{m_{2}}}$

Where $\tau_{\mathrm{m} 1}$ and $\tau_{\mathrm{m} 2}$ are time constants, $\boldsymbol{I}_{\boldsymbol{o}}$ is the peak value of the transient waveform, and the normalization factor $\eta$ is given by

$$
\eta_{m}=e^{-\left(\tau_{m 1} / \tau_{m 2}\right)\left(n_{m} \tau_{m 2} / \tau_{m 1}\right)^{1 / n_{m}}}
$$

Substituting the channel current waveform given by equations 1,2, and 3 into Maxwell equations to yield the radiated fields for $\boldsymbol{t} \geq \boldsymbol{D} / \boldsymbol{c}$ and at a horizontal distance D from the lightning channel base as

$$
\vec{H}(D, t)=\frac{v}{2 \pi c D} i(t-D / c) \hat{\phi}, \quad \vec{E}(D, t)=-\frac{\mu_{o} v}{2 \pi D} i(t-D / c) \hat{z}
$$

\section{THE TRANSMISSION LINE RESPONSE DUE TO EMP FIELDS COUPLING}

Transmission line model is used to study the EMP fields coupling to a line over or buried in the earth. The excitation fields are determined from the general expressions of the incident, reflected and transmitted plane waves. The induced distributed and lumped sources are then calculated and substituted into the transmission line (telegrapher) equations to yield the frequency and time-domain responses.

\section{A. The Excitation Fields}

An arbitrarily polarized plane wave of magnitude $\boldsymbol{E}_{\boldsymbol{o}}$, angles of incidence $(\boldsymbol{\psi}, \boldsymbol{\varphi})$ and polarization angle $\boldsymbol{\alpha}_{p}$ is incident on an imperfectly conducting half-space (earth with dielectric constant $\varepsilon_{r}$ and conductivity $\sigma_{g}$ ). For lines above the earth, the excitation fields are the summation of the incident and reflected. The reflected fields are related to the incident fields through Fresnel reflection coefficients $\boldsymbol{R}_{\boldsymbol{v}}$ and $\boldsymbol{R}_{\boldsymbol{h}}$ for vertically and horizontally polarized fields; respectively. These reflection coefficients are given by

$$
\begin{aligned}
& R_{v}=\frac{\varepsilon_{r}\left(1+\sigma_{g} / j \omega \varepsilon_{r} \varepsilon_{o}\right) \sin \psi-\left[\varepsilon_{r}\left(1+\sigma_{g} / j \omega \varepsilon_{r} \varepsilon_{o}\right)-\cos ^{2} \psi\right]^{1 / 2}}{\varepsilon_{r}\left(1+\sigma_{g} / j \omega \varepsilon_{r} \varepsilon_{o}\right) \sin \psi+\left[\varepsilon_{r}\left(1+\sigma_{g} / j \omega \varepsilon_{r} \varepsilon_{o}\right)-\cos ^{2} \psi\right]^{1 / 2}} \\
& R_{h}=\frac{\sin \psi-\left[\varepsilon_{r}\left(1+\sigma_{g} / j \omega \varepsilon_{r} \varepsilon_{o}\right)-\cos ^{2} \psi\right]^{1 / 2}}{\sin \psi+\left[\varepsilon_{r}\left(1+\sigma_{g} / j \omega \varepsilon_{r} \varepsilon_{o}\right)-\cos ^{2} \psi\right]^{1 / 2}}
\end{aligned}
$$


For buried lines in the earth, the excitation fields are simply the transmitted fields, which are related to the incident fields by Fresnel transmission coefficients $\left(\boldsymbol{T}_{\boldsymbol{v}}, \boldsymbol{T}_{\boldsymbol{h}}\right)$. For vertically and horizontally polarized fields, $\boldsymbol{T}_{v}$ and $\boldsymbol{T}_{\boldsymbol{h}}$ are given by

$$
T_{v}=\frac{2 Z_{o g} \sin \psi}{Z_{o} \sin \psi+Z_{o g} \sin \psi_{t}}, \quad T_{h}=\frac{2 Z_{o g} \sin \psi}{Z_{o g} \sin \psi+Z_{o} \sin \psi_{t}}
$$

where $Z_{o g}=\sqrt{j \omega \mu_{o} /\left(\sigma_{g}+j \omega \varepsilon_{r} \varepsilon_{o}\right)}$ is the earth characteristic wave impedance, the transmission angle is $\psi_{t}=\sin ^{-1}\left(\sqrt{1+\left(\frac{k \cos \psi}{\gamma_{g}}\right)^{2}}\right)$, and the wave propagation constant in the earth is $\gamma_{g}=\sqrt{j \omega \mu_{o}\left(\sigma_{g}+j \omega \varepsilon_{r} \varepsilon_{o}\right)}$

\section{B. Derivation and Solution of Transmission Line Equations}

The first telegrapher equation is derived by applying stokes theorem over the first Maxwell curl equation $\left(\nabla \times \vec{E}=-\boldsymbol{j} \omega \mu_{\boldsymbol{o}} \overrightarrow{\boldsymbol{H}}\right)$ to give

$$
\frac{d V(x)}{d x}+Z^{\prime}(\omega) I(x)=V_{s}^{\prime}(x)
$$

where the distributed (per-unit-length) voltage source $\boldsymbol{V}_{s}(\boldsymbol{x})$ is given in terms of the excitation magnetic field $\boldsymbol{H}^{e x}$ as

$$
\begin{aligned}
& V_{s}^{\prime}(x)=-j \omega \mu_{o} \int_{-\infty}^{h} H_{y}^{e x}(x, z) d z= \\
& -j \omega \mu_{o}\left[\int_{-\infty}^{0} H_{y}^{t}(x, z) d z+\int_{0}^{h}\left(H_{y}{ }^{i n c}(x, z)+H_{y}^{r e f}(x, z)\right) d z\right]
\end{aligned}
$$

and the per-unit-length impedance is $Z^{\prime}(\omega)=j \omega L^{\prime}+Z_{g}^{\prime}+Z_{\omega}^{\prime}$ with the following perunit-length parameters: $L^{\prime}=\frac{\mu_{o}}{2 \pi} \operatorname{Ln}(2 h / a), \quad C^{\prime}=2 \pi \varepsilon / \operatorname{Ln}(2 h / a)$, $Z_{g}^{\prime}=\frac{j \omega \mu_{o}}{2 \pi} \operatorname{Ln}\left(\frac{1+\gamma_{g} h}{\gamma_{g} \boldsymbol{h}}\right)$ as quoted in [9], and the per-unit-length wire impedance $\boldsymbol{Z}_{\omega}^{\prime}$ is approximated by [10] at low frequency where $\left|\gamma_{\omega} a\right|<<1$ by $Z_{\omega}^{\prime}=\frac{1}{\pi a^{2} \sigma_{\omega}}+j \omega \frac{\mu_{o}}{8 \pi}$, and at high frequency where $\left|\gamma_{\omega} a\right|>>1$ by $Z_{\omega}^{\prime}=\frac{1+j}{2 \pi a} \sqrt{\frac{\omega \mu_{\omega}}{2 \sigma_{\omega}}}$, and the propagation constant for a wire with conductivity $\sigma_{\omega}$ and permittivity $\varepsilon_{\omega}$ is given by $\gamma_{\omega}=\sqrt{\boldsymbol{j} \omega \mu_{o}\left(\sigma_{\omega}+\boldsymbol{j} \omega \varepsilon_{\omega}\right)}$.

The second telegrapher equation is similarly derived by applying Stokes theorem on the second curl Maxwell equation $(\nabla \times \overrightarrow{\boldsymbol{H}}=\boldsymbol{j} \omega \varepsilon \overrightarrow{\boldsymbol{E}}+\overrightarrow{\boldsymbol{J}})$ to give

$$
\frac{d I(x)}{d x}+Y^{\prime} V(x)=I_{s}^{\prime}(x)
$$


where the line admittance is $Y^{\prime}=\left(\frac{1}{j \omega C^{\prime}}+\frac{1}{Y_{g}^{\prime}}\right)^{-1}, Z_{g}^{\prime} Y_{g}^{\prime}=\gamma_{g}{ }^{2}=j \omega \mu_{o}\left(\sigma_{g}+j \omega \varepsilon_{g}\right)$, and the distributed current source is given by

$$
\begin{aligned}
& I_{s}^{\prime}(x)=-Y^{\prime} \int_{-\infty}^{h} E_{z}^{e x}(x, z) d z= \\
& -Y^{\prime}\left[\int_{-\infty}^{0} E_{z}^{t}(x, z) d z+\int_{0}^{h}\left(E_{z}^{i n c}(x, z)+E_{z}^{r e f}(x, z)\right) d z\right]
\end{aligned}
$$

The transmission line equations (9) and (11) are solved numerically to yield the line response $\boldsymbol{V}(\boldsymbol{x})$ and $\boldsymbol{I}(\boldsymbol{x})$ in the frequency domain, and using the inverse Fourier transform, one can get the time-domain response.

\section{NUMERICAL RESULTS AND DISCUSSIONS}

The transient waveform of the double exponential EMP specified by equation (1), where $E_{o}=50 \mathrm{kV} / \mathrm{m}, \alpha=\mathbf{4} \times \mathbf{1 0}^{6} \boldsymbol{S}^{-1}, \beta=\mathbf{4 . 7 6} \times 10^{8} S^{-1}$, is shown in Fig.1a, and its spectrum is displayed in Fig.1b. This EMP waveform is used to study the EMP fields interaction with electronic / electrical systems; specifically, the gun of a combat vehicle. The gun is simulated as a wire with length $\boldsymbol{L}=\mathbf{3} \boldsymbol{m}$, and radius $\boldsymbol{a}=\boldsymbol{8} \mathbf{c m}$. The wire is placed horizontally over an imperfect ground plane $\left(\varepsilon_{r}=\mathbf{1 0}, \sigma=\mathbf{0 . 0 0 2} \Omega^{-1}\right)$. The incident plane wave is vertically polarized $\left(\alpha_{p}=\mathbf{9 0}^{\circ}\right)$, as shown in Fig.2, where $\left(\psi=\mathbf{6 0}^{\circ}, \phi=\mathbf{0}^{\circ}\right)$. The short circuit current induced in the gun is calculated and its time-domain is plotted in Fig.3, where the results are compared satisfactorily with those obtained analytically by G. K. Deb as in [11] . The unnoticed discrepancy is probably because of the small gun length $(\boldsymbol{L}=\mathbf{3} \boldsymbol{m})$, and small length to height ratio $(\boldsymbol{L} / \boldsymbol{h}=\mathbf{1 . 5})$ used in transmission line modeling, which is accurate for larger dimensions.

The lightning channel model given by equations $2,3,4$, and 5 , is used to study the LEMP coupling problem. The lightning channel base current parameters are $I_{01}=10.7 \mathrm{kA}, \tau_{11}=0.25 \mu \mathrm{S}, \tau_{12}=2.5 \mu \mathrm{S}, I_{02}=10.7 \mathrm{kA}, \tau_{21}=0.25 \mu \mathrm{S}$,

$$
\tau_{22}=2.5 \mu \mathrm{S}, \mathrm{v}=1.3 \times 10^{8} \mathrm{~m} / \mathrm{S}, \alpha_{\mathrm{c}}=0.588 \mathrm{~km}^{-1}, \mathbf{n}_{1}=\mathbf{n}_{2}=2 .
$$

The transient waveform representing the lightning return - stroke current at the channel base, with the previously described parameters, is shown in Fig.4a, while its Fourier spectrum magnitude is shown in Fig.4b. The lightning channel is located at a distance $\boldsymbol{y}_{\boldsymbol{c}}=\mathbf{5 0} \boldsymbol{m}$ from the midpoint of a (matched) line with length $\boldsymbol{L}=\mathbf{1 0 0 0 m}$, radius $\boldsymbol{a}=\mathbf{5} \mathbf{m m}$, and located horizontally at $\boldsymbol{h}=\mathbf{1 0} \boldsymbol{m}$ above a perfect ground plane, as shown in Fig.5. The lightning induced voltage at either end of the line is calculated and plotted in Fig.6, where good coincidence is noticed with the results obtained by Nucci et al. [12]. A similar lightning channel current, but with current peak doubling is striking a telecommunication subscriber cable $(L=2000 \mathrm{~m}, a=0.5 \mathrm{~mm})$ at $\boldsymbol{h}=5 \mathrm{~m}$ above the lossy ground $\left(\varepsilon_{\mathrm{r}}=\mathbf{1 0}, \sigma=\mathbf{0 . 0 1} \Omega^{-1}\right)$. The line termination resistances are $Z_{1}=\mathbf{2 0 0} \Omega$, and $Z_{2}=10^{10} \Omega$. The induced voltage on the specified single line at termination $\boldsymbol{Z}_{\boldsymbol{l}}$ is calculated and shown in 
Fig.7, where coincidence is noticed with the results of Finite-Difference Time-Domain (FDTD) analysis [13].

The induced currents and voltages in cables by NEMP or LEMP, which are shown in figures 3,6 , and 7 would obviously be detrimental to many electronic components served by such cables, as is apparent from Table 1. Prevention of such damaging currents and voltages from affecting sensitive electronic components is the objective of EMP protection measures.

Table 1 Threshold damage energy to solid state components with $(1 \mu \mathrm{S})$ pulse [2]

\begin{tabular}{|c|c|}
\hline Solid-state Component & Damage Energy $[\mu \mathrm{J}]$ \\
\hline Shottky barrier diodes & $5: 70$ \\
\hline Silicon FETs & $10: 40$ \\
\hline Germanium transistors & $7: 300$ \\
\hline Silicon transistors & $10: 7000$ \\
\hline Integrated circuits & $10: 700$ \\
\hline Switching diodes & $7: 1000$ \\
\hline
\end{tabular}

\section{CONCLUSION}

Experimental waveforms of NEMP and LEMP have been studied, compared, and analyzed in time and frequency domains. The radiated EMP fields have also been derived. Based on the transmission line (telegrapher) equations, a numerical procedure has been developed to study EMP coupling with electrical / electronic systems. The proposed procedure has been applied to study the EMP interaction with practical configurations such as the gun of a combat vehicle illuminated by NEMP, an overhead power line, and a telecommunication subscriber cable immersed in lightning EMP fields. The transient and frequency responses of the lines above lossy ground have been evaluated in terms of induced voltages and currents. The results of the proposed procedure have been compared favorably with other analytical and numerical techniques such as FDTD. The proposed procedure can be applied straightforward for buried cables. Moreover, it is also suitable for EMP coupling analysis with multi - conductor lines by using the equivalent radius concept.

\section{REFERENCES}

[1] C. L. Longmire, "On the electromagnetic pulse produced by nuclear explosions," IEEE Trans. Electromagn. Compat., vol. EMC-20, pp. 3-13, Feb. 1978.

[2] K. S. H. Lee, Ed., "EMP Interaction: Principles, Techniques, and Reference Data," Washington, DC: Hemisphere, 1986.

[3] Handel, P.H., "Polarization Catastrophe Theory of Cloud Electricity Speculation on a New Mechanism for Thunderstorm Electrification," Journal of Geophysical Research, Vol.9, No.3, pp. 5857-5863, June 1985.

[4] Smith, A. A., Jr., "Coupling of External Electromagnetic Fields to Transmission Lines," Wiley, New York, 1977. 
[5] Taylor, C. D., R. S. Satterwhite, and C. W. Harrison, "The Response of a Terminated Two-Wire Transmission Line Excited by a Nonuniform Electromagnetic Field," IEEE Trans. Antennas Propag., Vol. AP-13, No.6, pp. 987-989, Nov. 1987.

[6] Agrawal, A. K., et al., "Transient response of Multiconductor Transmission Lines Excited by a Nonuniform Electromagnetic Field," IEEE Trans. Electromagn. Compat., Vol. EMC-22, No.2, May 1980.

[7] Rachidi, F., "Formulation of Field-to-Transmission Line Coupling Equations in Terms of Magnetic Excitation Field," IEEE Trans. Electromagn. Compat., Vol. EMC-35, No.3, August 1993.

[8] Uman, M. A., “The Lightning Discharge,” Academic Press, Inc., New York, 1987.

[9] Sunde, E. D., "Earth Conduction Effects in Transmission Systems," Van Nostrand, New York, 1949.

[10] Vance E. F., “Coupling to Shielded Cables,” Melbourne, FL, 1988.

[11] G. K. Deb, "Nuclear Electromagnetic Pulse Penetration Analysis in Combat Vehicle," IEEE Antennas \& Propagation Symposium, Vol.1, pp. 427-430, 2000.

[12] Nucci, C. A., et al., "Lightning-Induced Voltages on Overhead Lines," IEEE Trans. Electromagn. Compat., Vol. EMC-35, No.1, February 1993.

[13] Jae Cheol, Hyun Young Lee, Ga Jae Lim, Dong Chul Park, "FDTD Analysis on a Telecommunication Cable Immersed in Lightning EM Fields," IEEE Antennas \& Propagation Symposium, Vol.2, pp. 795-799, 2000.

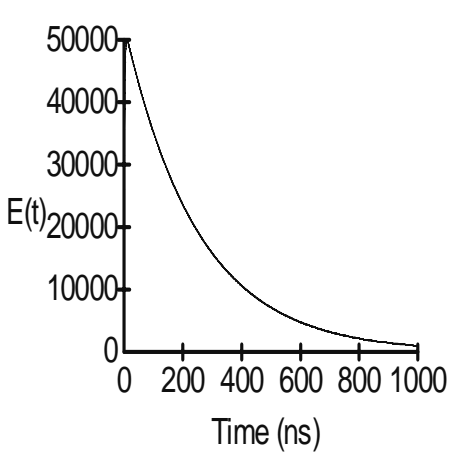

(a)Transient response

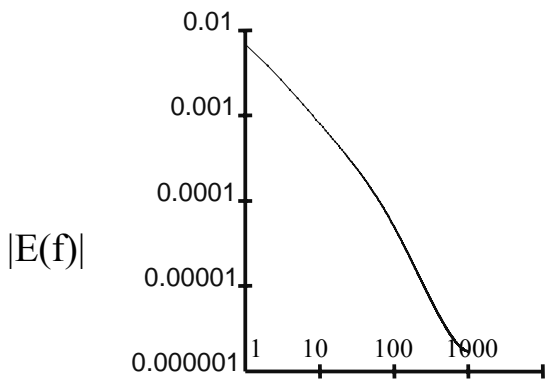

Frequency $(\mathrm{MHz})$

Fig.1. NEMP waveform, (a) time-domain, and (b) NEMP spectrum 


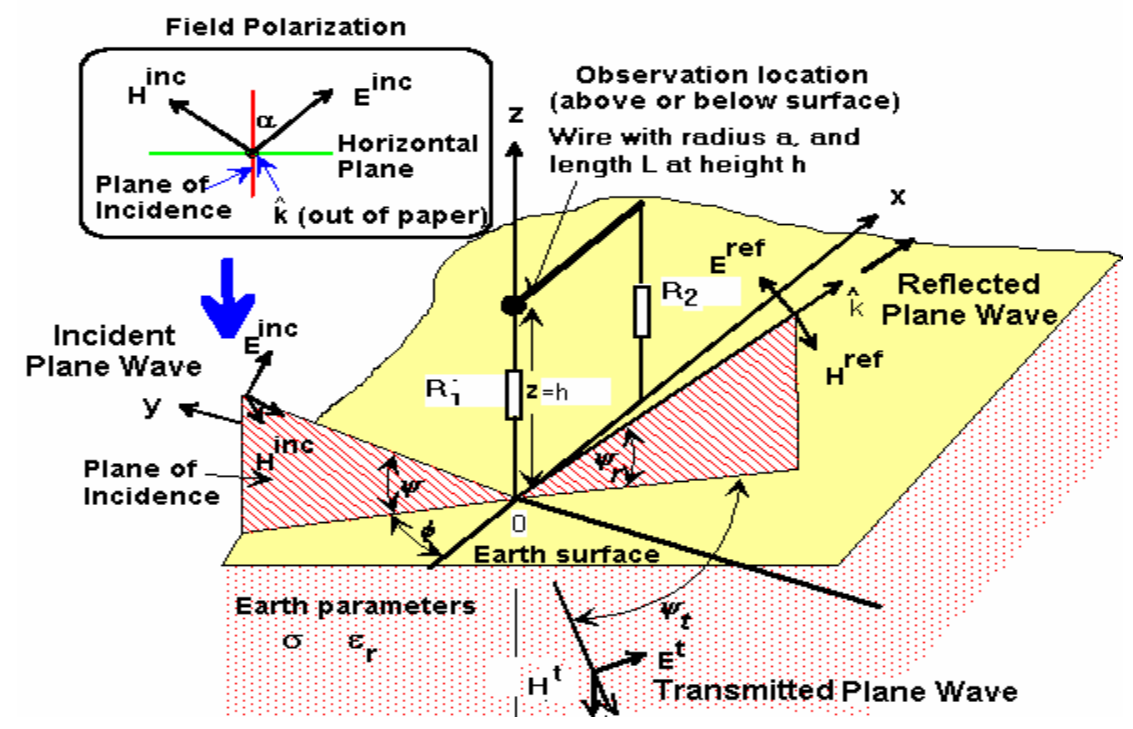

Fig.2. Geometry of (NEMP) electromagnetic fields interaction with a transmission line over a lossy ground plane.

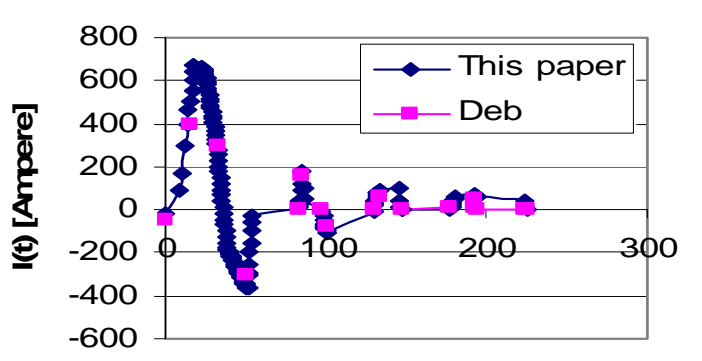

Time [nano sec.]

Fig.3. Calculated short circuit current induced at the point of the entry of the gun due to the NEMP waveform compared with results of G. K. Deb [11].

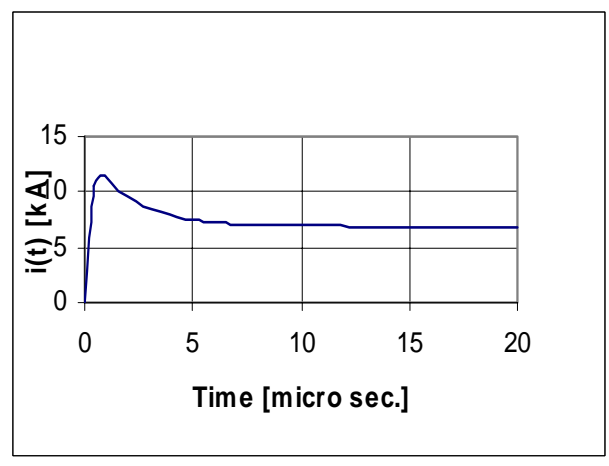

(a) LEMP transient waveform

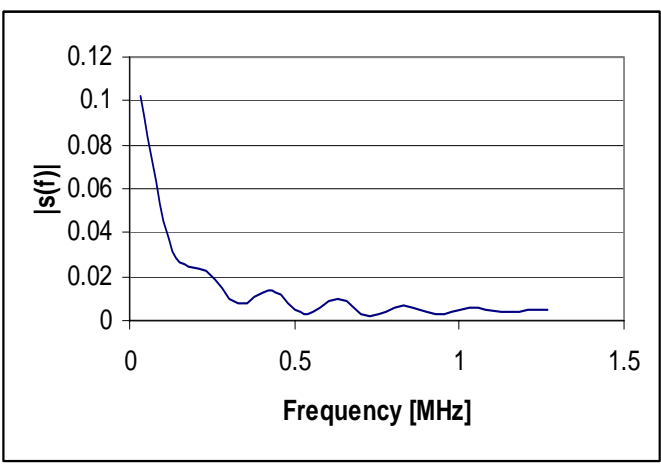

(b) LEMP spectrum

Fig.4. LEMP waveform, (a) time-domain, and (b) frequency-domain 


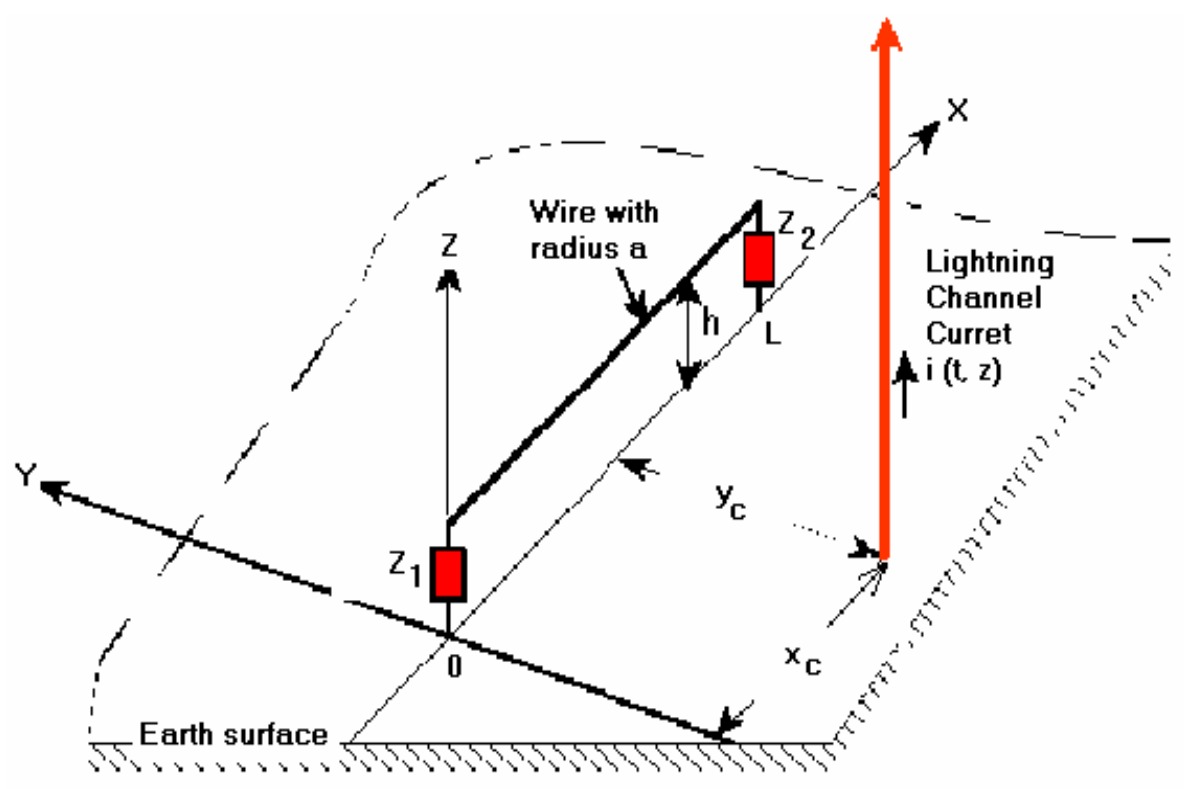

Fig.5. Lightning coupling geometry

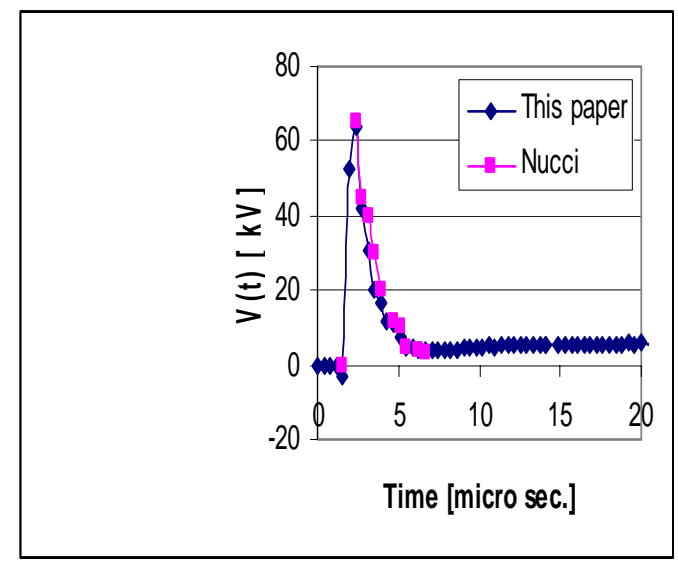

Fig.6. Lightning induced voltage at either end of an overhead power line, compared with Nucci [12]

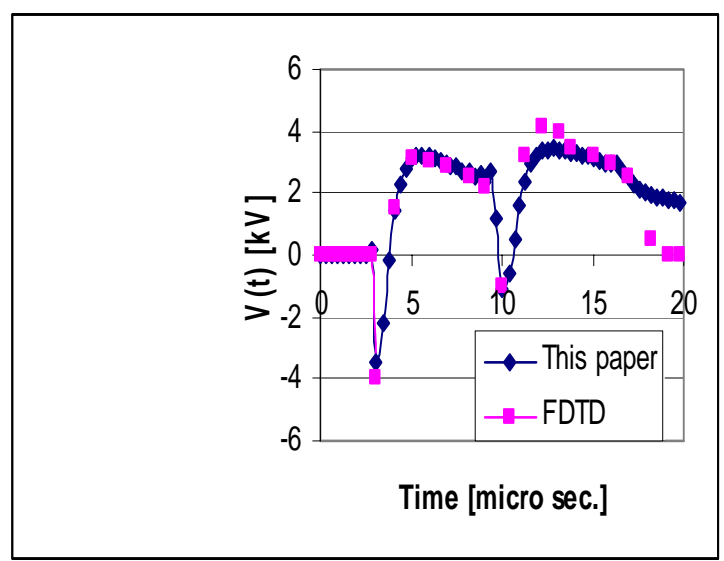

Fig.7. Lightning induced voltage at $\mathrm{Z}_{1}$ of a telecommunication cable, compared with FDTD analysis [13] 